\title{
Heavy ion collisions and black hole dynamics
}

\author{
Steven S. Gubser
}

Published online: 3 July 2007

(C) Springer Science+Business Media, LLC 2007

\begin{abstract}
Relativistic heavy ion collisions create a strongly coupled quark-gluon plasma. Some of the plasma's properties can be approximately understood in terms of a dual black hole. These properties include shear viscosity, thermalization time, and drag force on heavy quarks. They are hard to calculate from first principles in QCD. Extracting predictions about quark-gluon plasmas from dual black holes mostly involves solving Einstein's equations and classical string equations of motion. AdS/CFT provides a translation from gravitational calculations to gauge theory predictions. The gauge theory to which the predictions apply is $\mathcal{N}=4$ super-Yang-Mills theory. QCD is different in many respects from super-Yang-Mills, but it seems that its high temperature properties are similar enough to make some meaningful comparisons.
\end{abstract}

The Relativistic Heavy Ion Collider (RHIC) at Brookhaven National Laboratory collides gold nuclei at a total center of mass energy of $39 \mathrm{TeV}$. When the nuclei collide, a quark-gluon plasma (QGP) is formed. The QGP probably thermalizes before it blows itself apart. Its peak temperature is about $300 \mathrm{MeV}$. This is hotter than the QCD transition temperature, which is about $170 \mathrm{MeV}$. The physics of the QGP is described by quantum chromodynamics (QCD). But QCD is hard to solve. The reliability of perturbative methods is questionable because $\alpha_{s}$ may be as large as $1 / 2$ at the scale of RHIC physics. Lattice methods are well-suited for computing static quantities like pressure and entropy, but transport coefficients like shear viscocity and diffusion constants are hard to extract from the lattice because they relate to real-time processes rather than periodic Euclidean time.

Third Award in the 2007 Essay Competition of the Gravity Research Foundation.

S. S. Gubser $(\varangle)$

Joseph Henry Laboratories, Princeton University, Princeton, NJ 08544, USA

e-mail: ssgubser@Princeton.EDU 
The experimental summaries [1-4] provide a point of entry into the enormous literature on RHIC physics. A review of lattice results can be found in [5], and reviews of theoretical developments include [6-10].

The world of heavy ion physics was shaken by a result combining black hole physics and string theory $[11,12]$ :

$$
\frac{\eta}{s}=\frac{\hbar}{4 \pi k_{B}},
$$

where $\eta$ is the shear viscosity of a wide class of spatially extended black hole horizons and $s$ is the entropy density. (Usually I will set $\hbar=c=k_{B}=1$.) The result (1) is smaller by more than an order of magnitude than predicted for the QGP by perturbative QCD [13-16]. Experimental constraints from RHIC may be roughly summarized as $\eta / s \lesssim 0.3[15,17]$.

We are thus led to inquire $[18,19]$ : Can the QGP be described in terms of a dual black hole?

What hope there is for a positive answer comes from the AdS/CFT ${ }^{1}$ correspondence [20-22]. It says that $\mathcal{N}=4$ super-Yang-Mills (SYM) theory in four dimensions is dual to type IIB string theory on $A d S_{5} \times S^{5}$. (There will be no reason to keep track of the $S^{5}$. It relates to a global flavor symmetry in SYM.) Einstein gravity is a good description of the dynamics in $A d S_{5}$ - and, indirectly, the dual gauge theory- to the extent that the number of colors $N$ and the 't Hooft coupling $g_{\mathrm{YM}}^{2} N$ are both large. The success of the relation (1) suggests that, at least in one important respect, the QGP is more similar to strongly coupled SYM than to a weakly coupled plasma of quarks and gluons.

The result (1) is meaningful only when a hydrodynamical description of the QGP is valid, which is to say for times later than the thermalization time $\tau_{\text {therm }}$. Successful hydrodynamical models of RHIC physics assume $\tau_{\text {therm }} \approx 0.6-1.0 \mathrm{fm} / c[2,23$, 24 ]. But perturbative QCD calculations [24-26] predict $\tau_{\text {therm }} \gtrsim 2.5 \mathrm{fm} / c$. AdS/CFT encodes in black holes everything there is to know about thermal SYM. So it must make a prediction about $\tau_{\text {therm }}$. To extract this prediction, my students and I studied perturbations of the global $A d S_{5}$-Schwarzschild solution [27]. A conformal transformation shows that the unperturbed solution is dual to a radially expanding flow of thermal SYM matter. Comparing to the radial component of the collective flows in the QGP requires us to take the horizon radius $\rho_{H}$ of the black hole much bigger than the curvature scale $L$ of $A d S_{5}: \rho_{H} / L \sim 13$. The reason is that $\rho_{H} / L$ is roughly equated with the peak temperature in the gauge theory (about $300 \mathrm{MeV}$ ) times the extent of the thermal matter at maximal compression (about $10 \mathrm{fm}$ ). When $\rho_{H} / L \gg 1$, the perturbations split into two groups [27,28]: fast and slow quasi-normal modes. The slow modes can be approximately described in terms of solutions of the linearized Navier-Stokes equations in the boundary theory. They damp out slowly because the viscosity is small. The fast modes damp out to $1 / e$ of their original amplitude in a time no greater than

$$
\tau_{\text {fast }}=\frac{1}{8.6 T_{\text {peak }}} \approx 0.08 \mathrm{fm} / c .
$$

\footnotetext{
1 AdS stands for anti-de Sitter space, and CFT stands for conformal field theory.
} 


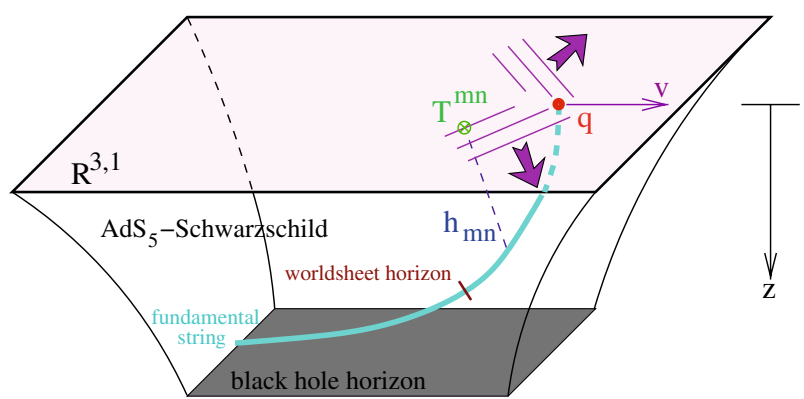

Fig. 1 A string trails behind a heavy quark $q$ into $A d S_{5}$-Schwarzschild. The quark creates a wake of stress-energy $\left\langle T^{m n}\right\rangle$ which is dual to gravitons $h_{m n}$ sourced by the string [29]

The first equality in (2) comes from solving the linearized Einstein equations for the slowest of the fast (non-hydrodynamic) modes. $T_{\text {peak }}$ is the peak temperature in the gauge theory. It is related to the Hawking temperature of the black hole. To obtain the last expression in (2) we used $T_{\text {peak }} \approx 300 \mathrm{MeV}$. Given the highly anisotropic momentum space distribution expected in the early stages of a RHIC collision, it is reasonable to expect that several $e$-folding times of the relevant thermalization processes must elapse before hydrodynamic approximations can be used. Thus one may estimate

$$
\tau_{\text {therm }} \sim 4 \tau_{\text {fast }} \approx 0.3 \mathrm{fm} / c .
$$

This estimate is risky because quasi-normal modes describe only the late-time stages of thermalization. However, when matched against estimates of $\tau_{\text {therm }}$ from plasma instabilities [24], the estimate (3) compares reasonably well, both in terms of precision and tenability of the final answer in the face of data.

$\mathcal{N}=4$ SYM does not have light fundamental quarks like QCD does. However, one can introduce infinitely massive quarks by dangling a string into translationallyinvariant $\mathrm{AdS}_{5}$-Schwarzschild, see Fig. 1.

The drag force on such a quark moving at a velocity $\vec{v}$ relative to the QGP is $[30,31]$

$$
\vec{F}=-\frac{\pi \sqrt{g_{\mathrm{YM}}^{2} N}}{2} T^{2} \frac{\vec{v}}{\sqrt{1-v^{2}}} .
$$

To understand the origin of (4), consider first the $A d S_{5}$-Schwarzschild geometry:

$$
d s^{2}=\frac{L^{2}}{z^{2}}\left(-h(z) d t^{2}+d \vec{x}^{2}+\frac{d z^{2}}{h(z)}\right) \quad \text { where } h=1-\frac{z^{4}}{z_{H}^{4}} .
$$

A steady-state configuration of the string must move with the quark:

$$
x^{1}=v t+\xi(z) \text { if } \vec{v}=(v, 0,0) .
$$

$\xi(z)$ measures how much the string trails behind the quark at a depth $z$. It can be determined explicitly by solving the string equations of motion, subject to a boundary 
condition at a causal horizon on the string worldsheet at $z_{*}=z_{H} \sqrt[4]{1-v^{2}}$. No signal can propagate classically along the string from $z>z_{*}$ to $z<z_{*}$. The drag force (4) can be computed from the flow of energy-momentum down the string.

To compare with data, consider $b$ and $c$ quarks, whose masses are well above characteristic RHIC temperatures. In [32] I argued that, with a physically motivated choice of $g_{\mathrm{YM}}^{2} N$, the result (4) translates (for energy densities characteristic of RHIC physics) into a time $t_{c} \approx 2.1 \mathrm{fm} / c$ for a charm quark's velocity relative to the QGP to fall by $1 / e$. Perturbative QCD estimates vary, but according to [33], $t_{c} \gtrsim 10 \mathrm{fm} / c$ is a representative range at energy densities typical of RHIC. Comparisons with data appear to favor models whose values for $t_{c}$ are closer to $4.5 \mathrm{fm} / c$ [32,34-36]. Determination of $t_{c}$ is complicated, however, by lack of an experimental tag to distinguish $c$ 's from $b$ 's, and by competing models of hadronization. In summary: perturbative predictions led to the incorrect expectation that charm quarks do not thermalize in RHIC collisions, whereas calculations from dual black holes are at least in the right ballpark in predicting that they do.

More physics can be extracted from the trailing string:

- The shape of the string encodes a configuration of color fields which interpolates smoothly between Coulombic near-field behavior and a sonic boom [29]. The stress tensor in the gauge theory is computed by solving linearized Einstein equations sourced by the string. Its Fourier-space components show rough agreement with jet-splitting data (see for example [37]), except that the Mach angle is about $15^{\circ}$ too small.

- The leading near-field correction to Coulombic behavior shows that energy density piles up in front of the quark for sufficiently relativistic velocities $v[38,39]$.

- There are stochastic fluctuations in the force on a heavy quark. These fluctuations can be related to properties of the worldsheet horizon [40-42]. They are an ana$\log$ of Hawking radiation. The fluctuations are stronger than the Einstein relation permits away from the non-relativistic regime $v \ll 1$, signalling that a Langevin description is inadequate. To capture the true stochastic dynamics, the quark and its near field should probably be treated as a composite object.

The dynamics of horizons is at the heart of every prediction made using AdS/CFT about quark-gluon plasmas. The Bekenstein-Hawking entropy-normalized against free field content, augmented by the leading stringy corrections, and evaluated at a reasonable value of $g_{\mathrm{YM}}^{2} N$-approximately matches lattice QCD calculations at RHIC energy scales $[5,32,43,44]$. Horizon dynamics interfaces spectacularly with hydrodynamics by predicting low viscosity, rapid thermalization, and sonic booms. The drag force calculation hinges on both the black hole horizon and the worldsheet horizon. But these horizons are not in our spacetime: they peak coyly at us from a fifth dimension employed by string theory to describe different energy scales.

To claim that the QGP is described by a dual black hole, we must be able to compute most (ideally all) of its measurable properties using AdS/CFT. This is a high bar. To clear it we probably have to use the holographic dual of a theory more closely resembling QCD than does $\mathcal{N}=4 \mathrm{SYM}$. Computations to date, however, give reason to hope. Agreement with data, while not spot-on, is approaching a level that is as good as I would expect given the significant differences between $\mathcal{N}=4 \mathrm{SYM}$ and QCD. 
While methods owing nothing to black hole physics continue — rightly— to dominate the theoretical literature on heavy ion collisions, I suspect we have only begun to learn what black holes can teach us about the ongoing experimental program at RHIC.

Acknowledgments I thank my students. This work was supported in part by the Department of Energy under Grant No. DE-FG02-91ER40671, and by the Sloan Foundation.

\section{References}

1. BRAHMS Collaboration, Arsene, I., et al.: Quark gluon plasma and color glass condensate at RHIC? The perspective from the BRAHMS experiment. Nucl. Phys. A757, 1-27 (2005). nucl-ex/0410020

2. PHENIX Collaboration, Adcox, K., et al.: Formation of dense partonic matter in relativistic nucleus nucleus collisions at RHIC: Experimental evaluation by the PHENIX collaboration. Nucl. Phys. A757, 184-283 (2005). nucl-ex/0410003

3. Back, B.B., et al.: The PHOBOS perspective on discoveries at RHIC. Nucl. Phys. A757, 28-101 (2005). nucl-ex/0410022

4. STAR Collaboration, Adams, J., et al.: Experimental and theoretical challenges in the search for the quark gluon plasma: the STAR collaboration's critical assessment of the evidence from RHIC collisions. Nucl. Phys. A757 102-183 (2005). nucl-ex/0501009

5. Karsch, F., Laermann, E.: Thermodynamics and in-medium hadron properties from lattice QCD. heplat/0305025

6. Shuryak, E.V.: Quantum chromodynamics and the theory of superdense matter. Phys. Rept. 61, 71-158 (1980)

7. Iancu, E., Leonidov, A., McLerran, L.: The colour glass condensate: an introduction. hep-ph/0202270

8. Kovner, A., Wiedemann, U.A.: Gluon radiation and parton energy loss. hep-ph/0304151

9. Kolb, P.F., Heinz, U.W.: Hydrodynamic description of ultrarelativistic heavy-ion collisions. nuclth/0305084

10. Shuryak, E.V.: What RHIC experiments and theory tell us about properties of quark-gluon plasma? Nucl. Phys. A750, 64-83 (2005). hep-ph/0405066

11. Policastro, E.V., Son, D.T., Starinets, A.O.: The shear viscosity of strongly coupled $N=4$ supersymmetric Yang-Mills plasma. Phys. Rev. Lett. 87, 081601 (2001). hep-th/0104066

12. Kovtun, P., Son, D.T., Starinets, A.O.: Viscosity in strongly interacting quantum field theories from black hole physics. Phys. Rev. Lett. 94, 111601 (2005). hep-th/0405231

13. Baym, G., Monien, H., Pethick, C.J., Ravenhall, D.G.: Transverse interactions and transport in relativistic quark-gluon and electromagnetic plasmas. Phys. Rev. Lett. 64, 1867-1870 (1990)

14. Arnold, P., Moore, G.D., Yaffe, L.G.: Transport coefficients in high temperature gauge theories. I: Leading-log results. JHEP 11, 001 (2000). hep-ph/0010177

15. Csernai, L.P., Kapusta, J.I., McLerran, L.D.: On the strongly-interacting low-viscosity matter created in relativistic nuclear collisions. Phys. Rev. Lett. 97, 152303 (2006). nucl-th/0604032

16. Huot, S.C., Jeon, S., Moore, G.D.: Shear viscosity in weakly coupled $N=4$ super Yang-Mills theory compared to QCD. hep-ph/0608062

17. Shuryak, E.: Emerging theory of strongly coupled quark-gluon plasma. hep-ph/0703208

18. Nastase, H.: The RHIC fireball as a dual black hole. hep-th/0501068

19. Shuryak, E., Sin, S.-J., Zahed, I.: A gravity dual of RHIC collisions. hep-th/0511199

20. Maldacena, J.M.: The large $N$ limit of superconformal field theories and supergravity. Adv. Theor. Math. Phys. 2, 231-252 (1998). hep-th/9711200

21. Gubser, S.S., Klebanov, I.R., Polyakov, A.M.: Gauge theory correlators from non-critical string theory. Phys. Lett. B428, 105-114 (1998). hep-th/9802109

22. Witten, E.: Anti-de Sitter space and holography. Adv. Theor. Math. Phys. 2, 253-291 (1998). hepth/9802150

23. Heinz, U.W.: Thermalization at RHIC. AIP Conf. Proc. 739, 163-180 (2005). nucl-th/0407067

24. Arnold, P., Lenaghan, J., Moore, G.D., Yaffe, L.G.: Apparent thermalization due to plasma instabilities in quark gluon plasma. Phys. Rev. Lett. 94, 072302 (2005). nucl-th/0409068

25. Baier, R., Mueller, A.H., Schiff, D., Son, D.T.: 'Bottom-up' thermalization in heavy ion collisions. Phys. Lett. B502, 51-58 (2001). hep-ph/0009237 
26. Molnar, D., Gyulassy, M.: Saturation of elliptic flow at RHIC: results from the covariant elastic parton cascade model MPC. Nucl. Phys. A697, 495-520 (2002). nucl-th/0104073

27. Friess, J.J., Gubser, S.S., Michalogiorgakis, G., Pufu, S.S.: Expanding plasmas and quasinormal modes of anti-de Sitter black holes. hep-th/0611005

28. Kovtun, P.K., Starinets, A.O.: Quasinormal modes and holography. Phys. Rev. D72, 086009 (2005). hep-th/0506184

29. Friess, J.J., Gubser, S.S. Michalogiorgakis, G., Pufu, S.S.: The stress tensor of a quark moving through $N=4$ thermal plasma. hep-th/0607022

30. Herzog, C.P., Karch, A., Kovtun, P., Kozcaz, C., Yaffe, L.G.: Energy loss of a heavy quark moving through $N=4$ supersymmetric Yang-Mills plasma. hep-th/0605158

31. Gubser, S.S.: Drag force in AdS/CFT. hep-th/0605182

32. Gubser, S.S.: Comparing the drag force on heavy quarks in $N=4$ super-Yang-Mills theory and QCD. hep-th/0611272

33. van Hees, H., Rapp, R.: Thermalization of heavy quarks in the quark-gluon plasma. Phys. Rev. C71, 034907 (2005). nucl-th/0412015

34. van Hees, H., Greco, V., Rapp, R.: Heavy-quark probes of the quark-gluon plasma at RHIC. Phys. Rev. C73, 034913 (2006). nucl-th/0508055

35. STAR Collaboration, Abelev, B.I., et al.: Transverse momentum and centrality dependence of high-p(T) non-photonic electron suppression in $\mathrm{Au}+\mathrm{Au}$ collisions at s(NN)**(1/2) $=200-\mathrm{GeV}$. nucl-ex/0607012

36. PHENIX Collaboration, Adare, A.: Energy loss and flow of heavy quarks in $\mathrm{Au}+\mathrm{Au}$ collisions at $\sqrt{s_{\mathrm{NN}}}=200 \mathrm{GeV}$. nucl-ex/0611018

37. PHENIX Collaboration, Adler, S.S., et al.: Modifications to di-jet hadron pair correlations in $\mathrm{Au}+\mathrm{Au}$ collisions at $\mathrm{s}(\mathrm{NN}) * *(1 / 2)=200-\mathrm{GeV}$. nucl-ex/0507004

38. Gubser, S.S., Pufu, S.S.: Master field treatment of metric perturbations sourced by the trailing string. hep-th/0703090

39. Yarom, A.: On the energy deposited by a quark moving in an $N=4$ SYM plasma. hep-th/0703095

40. Casalderrey-Solana, J., Teaney, D.: Heavy quark diffusion in strongly coupled $N=4$ Yang Mills. hep-ph/0605199

41. Gubser, S.S.: Momentum fluctuations of heavy quarks in the gauge-string duality. hep-th/0612143

42. Casalderrey-Solana, J., Teaney, D.: Transverse momentum broadening of a fast quark in a $N=4$ Yang Mills plasma. hep-th/0701123

43. Gubser, S.S., Klebanov, I.R., Peet, A.W.: Entropy and temperature of black 3-Branes. Phys. Rev. D54, 3915-3919 (1996). hep-th/9602135

44. Gubser, S.S., Klebanov, I.R., Tseytlin, A.A.: Coupling constant dependence in the thermodynamics of $N=4$ supersymmetric Yang-Mills theory. Nucl. Phys. B534, 202-222 (1998). hep-th/9805156 